\title{
RETENÇÃO PLACENTÁRIA E RUPTURA UTERINA EM ÉGUA - RELATO DE CASO
}

SANTOS, Fernanda Carlini Cunha dos ${ }^{1 ;}$
FEIJÓ, Lorena Soares ${ }^{1 ;}$
PAZINATO, Fernanda Maria ${ }^{1 ;}$
AMARAL, Lorena Alvariza ${ }^{2} ;$
MARTINS, Charles Ferreira ${ }^{2} ;$
NOGUEIRA, Carlos Eduardo Wayne ${ }^{2} ;$

${ }_{1}^{1}$ Programa de Pós-Graduação em Veterinária da UFPEL, ${ }^{2}$ Médico Veterinário, Professor Doutor da Faculdade de Veterinária da UFPEL.

\section{RESUMO}

A retenção placentária está relacionada à quadros de inércia uterina, podendo acarretar alterações sistêmicas. A ruptura uterina provoca contaminação da cavidade abdominal, induz o desencadeamento de peritonite, agravando o quadro clínico e representando risco a vida do animal. O objetivo deste trabalho foi relatar um caso de retenção placentária e ruptura uterina em uma égua. Uma égua, obesa, cinco dias após apresentar parto distócico, foi atendida sendo observado ao exame clínico taquicardia, taquipnéia, congestão de mucosa, febre e retenção placentária. À ultrassonografia, o útero estava distendido, com espessamento de parede uterina, conteúdo anecóico e pontos hiperecogênicos no lúmen. O hemograma apresentava leucocitose, neutrofilia, linfopenia e presença de bastonetes. A paracentese abdominal foi improdutiva. Foi iniciado tratamento com flunixin meglumine 1,1 mg/kg, penicilina G potássica $20.000 \mathrm{UI} / \mathrm{kg}$, gentamicina 6,6 $\mathrm{mg} / \mathrm{kg}$, oxitocina $20 \mathrm{UI}$, omeprazol $2 \mathrm{mg} / \mathrm{kg}$, heparina $60 \mathrm{UI} / \mathrm{kg}$, fluidoterapia e lavagem uterina. Após três dias de tratamento, o quadro clínico agravou-se e o animal evoluiu a óbito. Na necropsia foi visualizado intenso edema no subcutâneo da região abdominal, fígado com esteatose, úlceras gástricas, intensa deposição de fibrina na cavidade abdominal, ruptura uterina com segmento placentário em protrusão para cavidade abdominal, endométrio em necrose e presença de fragmentos placentários em autólise. A obesidade é sugerida como um fator de risco a ocorrência de distocia. A retenção placentária favoreceu a ocorrência de ruptura uterina, proporcionando o extravasamento do conteúdo uterino para a cavidade abdominal, sendo de difícil tratamento, evoluindo para óbito por peritonite e endotoxemia.

Palavras-chave: Distocia. Endotoxemia. Parto. Puerpério. 


\section{INTRODUÇÃO}

A gestação é o período compreendido entre a data da ovulação e o parto. A duração do período gestacional em éguas é de aproximadamente 330 a 345 dias, no entanto fisiologicamente pode apresentar grande variação (ROSSDALE, 1993). O parto geralmente ocorre no período noturno e pode ser dividido em três fases: a primeira fase denominada de prodrômica; a segunda fase caracterizada pela expulsão fetal; terceira a fase corresponde à expulsão das membranas fetais (CHRISTENSEN, 2011).

A retenção placentária é definida como a falha na expulsão de parte ou da totalidade da membrana alantocoriônica, com ou sem a membrana amniótica, num intervalo de até três horas após o parto (BLANCHARD et al., 2010). O parto distócico está associado a uma maior incidência de retenção placentária que o eutócico, assim como éguas que já tiveram um episódio de retenção são mais susceptíveis a reincidência (PROVENCHER et al., 1988).

A etiopatogenia da retenção placentária está relacionada diretamente a quadros de inércia uterina, e no caso de distocia a exaustão do miométrio, estiramento excessivo e a lesões na parede uterina. Lesões transmurais na parede uterina podem induzir a migração de bactérias e mediadores inflamatórios para cavidade abdominal, resultando em alterações sistêmicas. A laceração na totalidade da parede uterina induz a ocorrência de peritonite aguda, sendo que os sinais clínicos são observados 24 a 48 horas após a ocorrência desta alteração. 0 diagnóstico é dificultado pelo excessivo tamanho do útero no pós-parto imediato, sendo recomendada a palpação e ultrassonografia via retal, palpação vaginal, e em caso de sinais sugestivos de peritonite, a realização de hemograma e paracentese (DOLENTE, 2004). Sutter et al. (2003) constataram que o diagnóstico de laceração em corpo do útero foi realizado por via retal e vaginal em todas éguas avaliadas $(n=8)$, enquanto que o diagnóstico de laceração localizada em corno uterino só foi possível de ser realizado em $13 \%$ das éguas avaliadas $(n=22)$ pelos mesmos métodos, os $77 \%$ restantes foram observadas durante laparotomia exploratória. Os mesmos autores comentaram que a incidência de lesão em corno é maior que em corpo uterino, dificultando o diagnóstico precoce e adoção de medidas terapêuticas apropriadas. As lacerações e rupturas de útero são infrequentes, porém acarretam sérias complicações secundárias na égua e apresentam risco potencial de vida. 
Este trabalho tem como objetivo relatar o caso de uma égua com retenção placentária e laceração uterina.

\section{RELATO DE CASO}

Um equino, fêmea, raça Crioula, com sete anos de idade foi atendido em uma propriedade particular por um médico veterinário autônomo devido a um parto distócico. Os proprietários relataram que a égua estava com aproximadamente 330 dias de gestação, apresentava ingurgitamento de glândula mamária, era multípara, não possuía histórico de distocia prévia e estava em trabalho de parto há aproximadamente 24 horas, sem manipulação prévia. O feto estava morto e em apresentação longitudinal anterior posição dorsal com membros estendidos e pescoço flexionado. Nesta ocasião foi realizado auxílio ao parto através de reposicionamento e tração manual do feto. Após a manipulação obstétrica os proprietários relataram que não foi observada a expulsão das membranas fetais. Foi prescrita a administração de antibióticos, antiinflamatórios e ocitocina.

Aproximadamente cinco dias após a remoção do feto, a fêmea foi encaminhada ao Hospital de Clínicas Veterinária (HCV) da Universidade Federal de Pelotas (UFPEL) para atendimento, devido à piora no quadro clínico. Ao exame clínico, a égua apresentava consciência alerta, condição corporal classificada em 8 (escala 1-9) (HENNEKE et al., 1983), portanto obesa, frequência cardíaca de 80 bpm, frequência respiratória de 24 rpm, mucosas congestas, tempo de perfusão capilar de três segundos, temperatura retal de $38,6{ }^{\circ} \mathrm{C}$, auscultação abdominal com hipomotilidade intestinal em todos os quadrantes, discreta distensão abdominal e pulso digital aumentado em todos os membros. À ultrassonografia via transretal, o útero apresentava-se distendido, com espessamento de parede uterina, conteúdo anecóico e pontos hiperecogênicos dispersos em seu lúmen. Na avaliação da cavidade vaginal e vulva (Figura 1) foram observadas lacerações e áreas de necrose focal. 


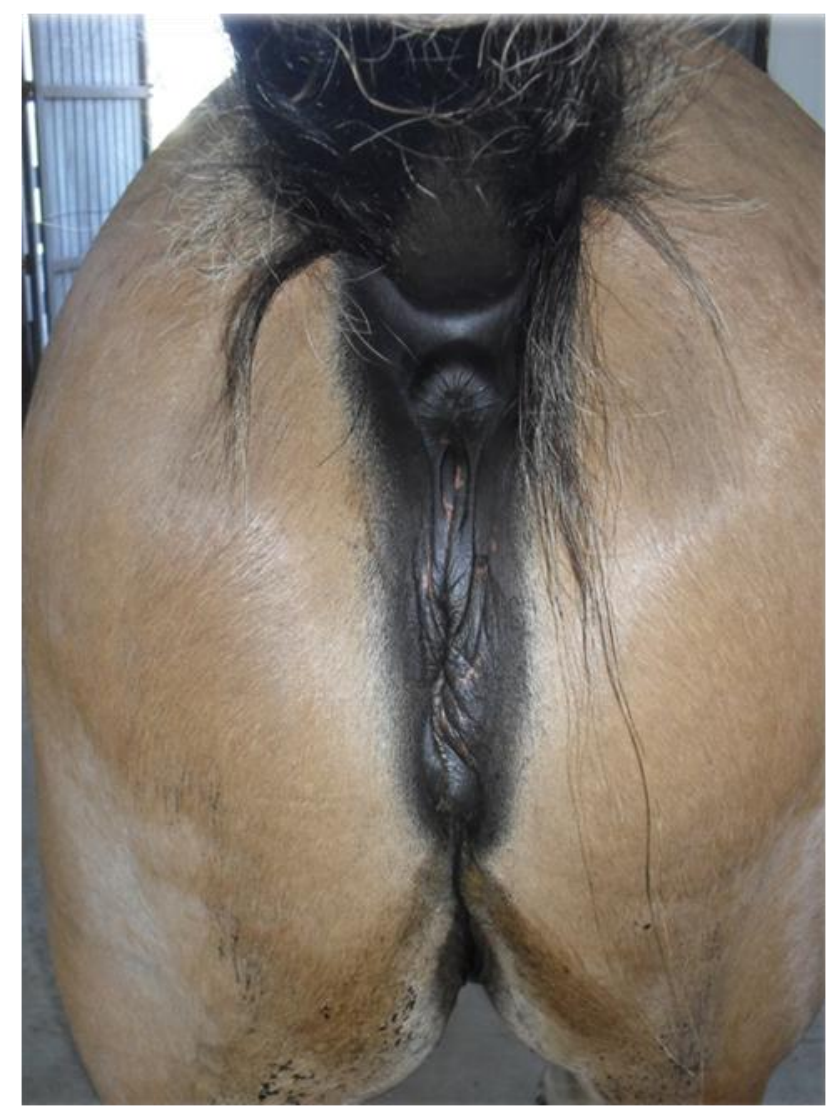

Figura 1 - Lacerações na vulva em uma égua Crioula devido ao parto distócico.

Na histeroscopia foi visualizada extensa área de placenta necrótica aderida ao endométrio além de acúmulo de líquido intrauterino de coloração amarronzada, turva e com debris celulares. Na avaliação hematológica foi constatada leucocitose $\left(13.200 \times 10^{3} / \mathrm{ul}\right)$ com neutrofilia $\left(8.844 \times 10^{3} / \mathrm{ul}\right)$, linfopenia $\left(2.376 \times 10^{3} / \mathrm{ul}\right)$ e presença de bastonetes $(1.848 \times$ $10^{3} / \mathrm{ul}$ ). Os valores hematimétricos (hemácias $7,7 \times 10^{6} / \mathrm{ul}$, hemoglobina $13,2 \mathrm{~g} / \mathrm{dl}$ e hematócrito 37\%) estavam dentro dos parâmetros de referência para espécie (GRONDIN; DEWITT, 2010). Foi realizada paracentese abdominal, com punções craniais e caudais a cicatriz umbilical, no entanto não foi obtido sucesso na colheita de líquido abdominal.

Após realização do exame clínico geral foi iniciado tratamento medicamentoso com antiinflamatório (flunixin meglumine 1,1 mg/kg IV, 12-12 h), antibióticos (penicilina G potássica $20.000 \mathrm{UI} / \mathrm{kg}$ IV, 6-6 h e gentamicina 6,6 mg/kg IM 24-24 h), agentes ecbólicos (oxitocina 20 UI IV, 6-6 h), protetor de mucosa gástrica (omeprazol 2 mg/kg VO, 24-24 h), heparinizante (heparina $60 \mathrm{UI} / \mathrm{kg} \mathrm{SC}$ ) e fluidoterapia com solução de Ringer com lactato, 
conforme a avaliação diária. A lavagem uterina foi realizada com água e iodo tópico a cada 12 horas. Durante a lavagem uterina foi constatada presença de conteúdo purulento em abundância, fétido, de coloração amarronzada, debris celulares e fragmentos de placenta em estado de autólise (Figura 2).

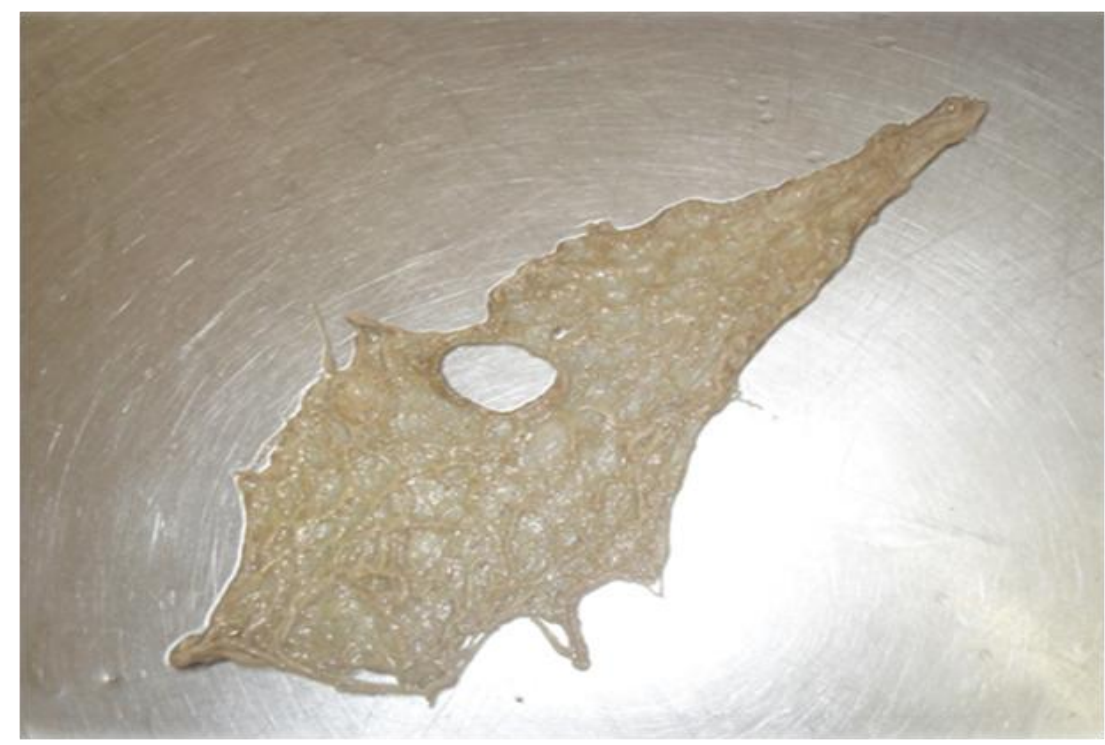

Figura 2 - Segmento placentário em autólise recuperado durante a lavagem uterina.

Durante o internamento, o animal foi alimentado com feno, pasto nativo e água ad libitum, apresentando redução no apetite. Três dias após a chegada ao HCV, à avaliação hematológica indicou aumento no quadro de leucocitose $\left(19.500 \times 10^{3} / \mathrm{ul}\right)$ com neutrofilia $\left(12.235 \times 10^{3} / \mathrm{ul}\right)$, linfopenia $\left(2.145 \times 10^{3} / \mathrm{ul}\right)$ e presença de bastonetes $\left(1.560 \times 10^{3} / \mathrm{ul}\right)$. Os valores hematimétricos apresentaram redução (hemácias 6 × 10\% $/ \mathrm{ul}$, hemoglobina $13 \mathrm{~g} / \mathrm{dl}$ e hematócrito 31\%), atribuído à hidratação do paciente.

O animal apresentava sensibilidade moderada à palpação externa da parede abdominal e distensão nesta região. Foi administrado fluidoterapia com solução de Ringer com lactato na tentativa de estabilização do quadro clínico do paciente, no entanto não foi obtida resposta terapêutica e o animal evoluiu para o óbito.

Na necropsia foi visualizado intenso edema no subcutâneo da região abdominal (cerca de 10 $\mathrm{cm}$ de espessura), rins com aspecto moteado, fígado com esteatose, úlceras gástricas, intensa deposição de fibrina na cavidade abdominal e em todos os órgãos, na avaliação histopatológica os rins apresentavam necrose tubular aguda. No útero foi visualizada uma 
ruptura de aproximadamente $8 \mathrm{~cm}$ de diâmetro entre o corpo uterino e o corno gravídico com segmento placentário em protrusão para cavidade abdominal (Figura 3), endométrio com extensa área de necrose, conteúdo fétido e presença de fragmentos placentários em estágio de autólise (Figura 4).

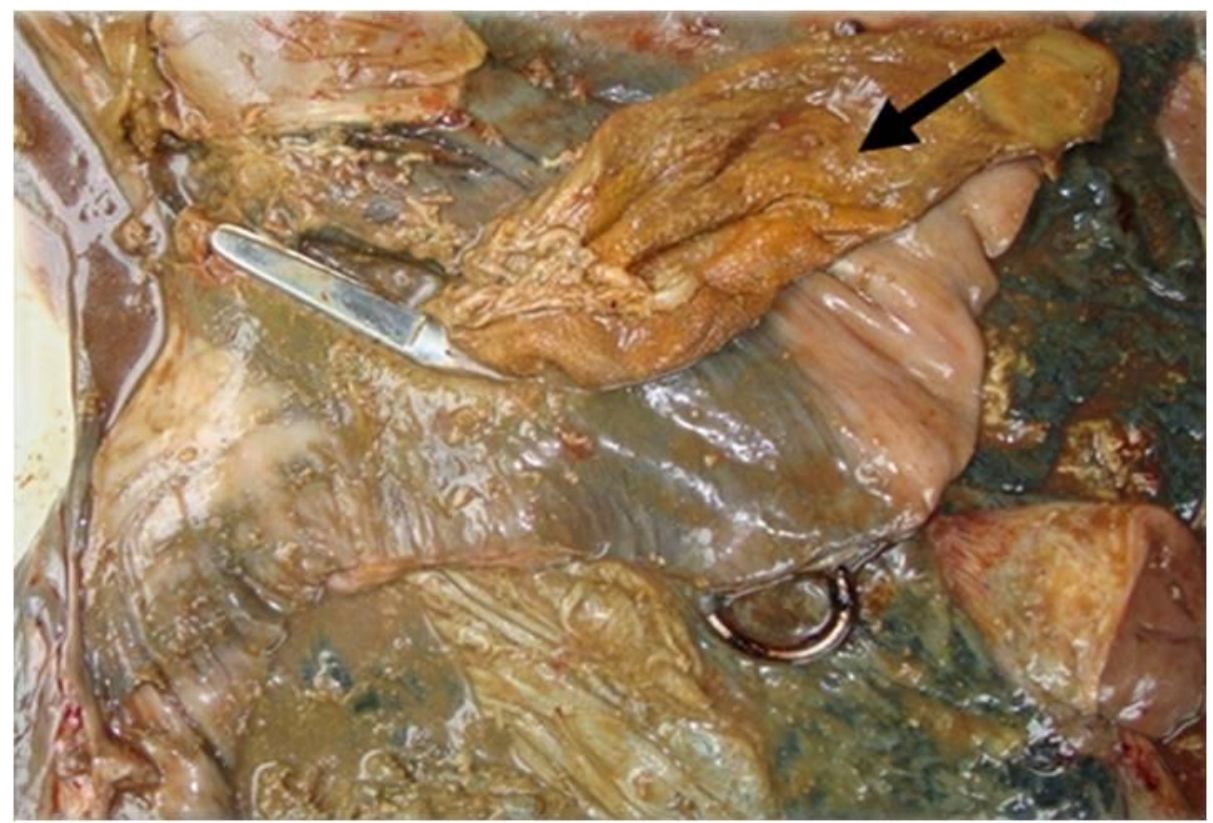

Figura 3 - Ruptura uterina de aproximadamente $8 \mathrm{~cm}$ de diâmetro entre corpo uterino e corno gravídico com segmento placentário (seta) em protrusão para cavidade abdominal.

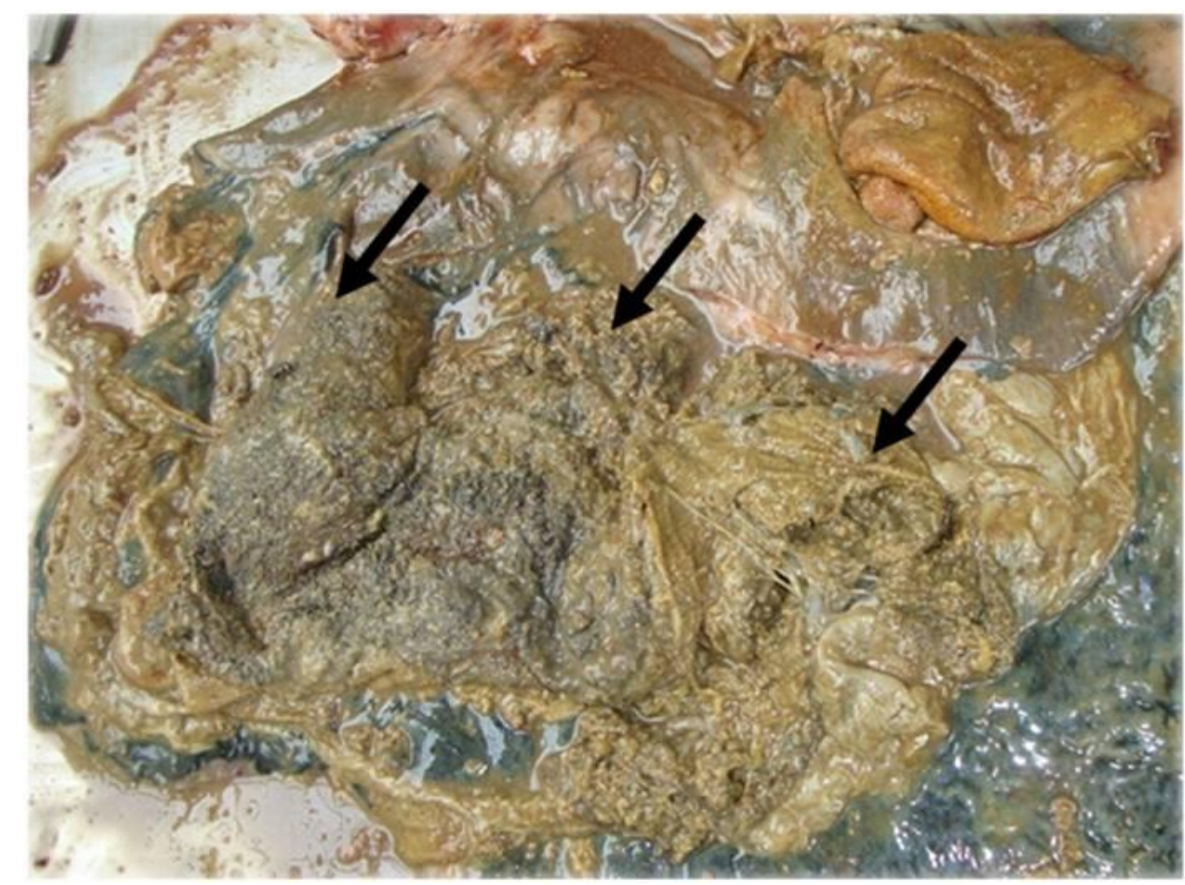

Figura 4 - Lúmen uterino em necrose (demarcado por setas). 


\section{RESULTADOS E DISCUSSÃO}

A incidência de distocia na espécie equina é baixa, embora apresente considerável variação conforme a raça. Equinos de tração apresentam maior incidência de partos distócicos de origem materna, uma vez que possuem musculatura bastante desenvolvida, ocasionando estreitamento físico do canal do parto (TIBARY et al., 2012).

O excessivo depósito de gordura corporal em equinos é um importante fator de risco no desenvolvimento de doenças como hiperlipidemia, hiperglicemia, resistência a insulina, síndrome de Cushing, esteatose hepática, podendo ocasionar alterações no sistema respiratório, lesões estrangulativas intestinais, queda do desempenho atlético, laminite (KEARNS et al., 2002) e queda na fertilidade (HENNEKE et al., 1983). No estado do Rio Grande do Sul, os cavalos Crioulos apresentam tendência ao sobrepeso, uma vez que por questões culturais e tradicionais, este padrão é bem visto pelos proprietários. Este conceito não é correto, tendo em vista as consequências da obesidade em animais destinados principalmente a competição e reprodução.

Em humanos, a obesidade provoca alterações no sistema cardíaco, pulmonar, endócrino e imunológico (CONWAY; RENÉ, 2004), além de aumentar a taxa de complicações no período pós-parto (RUGE; ANDERSEN, 1985) e risco de distocia (CHRISTOFFERSSON, 2002). A suscetibilidade de adquirir infecções, incapacidade em combatê-las e desenvolvimento de doenças crônicas inflamatórias são maiores em indivíduos obesos (ALVES, 2006). A obesidade foi relacionada à maior incidência de partos distócicos em bovinos (ZIMMERMAN; GREENE, 1971) e caninos (JOHNSON, 2008). Também há evidências que é uma doença inflamatória, (CARRASCOSA et al., 2014; EL-WAKKAD et al., 2013; ZORAN, 2010), sendo relevante este aspecto em pacientes críticos, como no presente caso com exacerbação da endotoxemia.

A principal complicação que ocorre no puerpério de fêmeas equinas é a retenção placentária, sendo que esta é determinada por falha na expulsão das membranas fetais em um período de 3 horas após o parto (BLANCHARD et al., 2010). A presença da placenta no lúmen uterino causa endometrite e metrite, atrasando a involução do órgão. 
Como terapia inicial, Turner (2007) propõe três regimes baseado no uso de ocitocina, sendo estes: administração de 10-20 UI IM ou IV a cada 15 minutos, durante 2 horas pela manhã e 2 horas à tarde; 10-20 UI IM ou IV a cada hora, durante 24 horas; infusão endovenosa contínua de ocitocina iniciando com dose de $1 \mathrm{UI}$ por minuto dissolvida em solução de Ringer com lactato. A utilização de ocitocina em bolus provoca contrações uterinas em curto intervalo de tempo, no entanto geralmente são suficientes na resolução de casos não complicados. Turner (2007) também relata que a administração exógena de ocitocina não provoca a desunião das membranas fetais num espaço de 12 horas, então é comum estas permanecerem retidas por vários dias até necrosarem e descolarem do endométrio.

Nos casos refratários a ocitocina, a prostaglandina representa outra opção, uma vez que este hormônio possui efeito uterotônico mais prolongado (LE BLANC et al., 1994). No entanto, durante um processo inflamatório há aumento na produção de prostaglandinas, sendo que a administração exógena apresenta potencial risco de exacerbação do quadro em pacientes com sinais de resposta inflamatória sistêmica. Mediadores lipídicos, como a prostaglandina, participam do processo de vasodilatação, aumento da permeabilidade vascular, sensibilizam os nociceptores e estimulam os centros hipotalâmicos de termorregulação (JÚNIOR et al., 2007), não sendo utilizados no presente relato em virtude das alterações sistêmicas. A carbetocina também é um estimulante de tonicidade uterina com ação prolongada, no entanto ainda há escassos trabalhos científicos comprovando sua ação para esta patologia na espécie equina (TURNER, 2007), apesar de sua frequente utilização empírica. A placenta em estado de autólise fica friável e aderida ao epitélio uterino, sendo frequentemente necessária a correção de causa primária e associação de tratamentos, através de procedimentos e terapia medicamentosa.

A lavagem do útero tem como intuito a remoção de bactérias e resíduos do lúmen uterino, favorecendo a regeneração e involução do órgão. Threlfall (2007) ressalta que a manipulação do órgão aumenta a circulação local e consequentemente aumenta a absorção de substâncias do lúmen uterino para o sistema circulatório, podendo agravar alterações sistêmicas, como a endotoxemia. Ao preencher o útero com água, os receptores de distensão visceral são ativados seguindo-se da liberação endógena de ocitocina e a 
separação das microvilosidades das criptas endometriais (HUDSON et al., 2005). Para realização do procedimento, Vandeplassche et al. (1983) têm como protocolo a utilização de 50-100 litros de água em fluxo contínuo, contudo Brinsko (2001) recomenda o uso de 12 litros de água morna com $5 \mathrm{~mL}$ de solução de iodo-povidona e 8,5 g de sal por litro, sendo esta última opção mais relevante pela osmolaridade da solução, não sendo agressiva ao ambiente uterino. A lavagem uterina do presente caso foi realizada com grande quantidade de água com iodo tópico, devido ao excessivo tamanho do órgão, abundante quantidade de anexos fetais e custo do tratamento. Apesar da manipulação uterina representar risco potencial de agravamento no quadro sistêmico, a negligência na limpeza deste órgão favorece o acúmulo de restos fetais e proliferação de microrganismos patogênicos. A multiplicação destes microorganismos patogênicos no lúmen uterino é mais prejudicial do que o risco de aumento na circulação sanguíneo devido manipulação do trato reprodutivo, com consequente migração bacteriana e agravamento da condição sistêmica.

Em relação à remoção manual da placenta, esta é somente recomendada nos casos em que o descolamento da mesma estiver em estágio avançado e quando a operação é rápida e não acompanhada de hemorragia (HORTA, 2000). Os principais distúrbios atribuídos à remoção manual da placenta são hemorragia, retardo na involução uterina, endometrite, cérvix permanecendo aberta por longo período e dano permanente ao endométrio devido à manipulação resultando em redução na fertilidade (THRELFALL, 2007). No entanto, CuervoArango e Newcombe (2009) constataram que remoção manual da placenta no pós-parto imediato não apresentou efeito negativo na fertilidade e parâmetros reprodutivos das fêmeas estudadas. Após longo período de retenção, como no presente caso, a placenta torna-se necrosada, fragmentada e de difícil manipulação, não favorecendo a tração da mesma e aumentando o risco de dano permanente ao endométrio.

Em relação à laceração uterina, Fischer e Philips (1986) relataram a reparação cirúrgica em duas éguas, obtendo índices de fertilidade aceitáveis nos períodos reprodutivos subsequentes. No presente caso, laceração/ruptura uterina pode ter ocorrido durante a manipulação obstétrica e associadas às alterações sistêmicas esta lesão foi gradativamente aumentando em tamanho, com extravasamento de conteúdo para cavidade abdominal. 
Caso a ruptura seja detectada de forma precoce o tratamento de eleição é a correção cirúrgica, sendo o prognóstico relacionado principalmente a extensão da lesão, tempo de evolução e contaminação da cavidade abdominal, culminando em peritonite. A obtenção do líquido peritoneal por meio da paracentese abdominal, considerada uma prática fácil, segura e de baixo custo, é o teste laboratorial mais esclarecedor para auxiliar a classificação do tipo de doença e também para determinar a severidade da lesão abdominal (TULLENERS, 1983). Isto torna a análise de líquido peritoneal um exame importante e útil não só para o diagnóstico, mas também para o prognóstico e direcionamento da conduta clínica em equinos (MESSER, 1995). No presente caso a realização de paracentese foi improdutiva devido à obesidade e edema subcutâneo localizado na região abdominal, visualizado durante a necropsia, no entanto devido ao histórico do animal e condições clínicas a ocorrência de peritonite e endotoxemia são um achado comum. A peritonite ocorreu no presente caso devido à contaminação da cavidade abdominal por conteúdo intrauterino, sendo que o prognóstico para esta enfermidade é sempre de reservado a desfavorável, com mortalidade variando de 30-67\% (HANSON, 1999). Sem a remoção da causa primária a chance de resolução do processo é mínima devido à constante entrada de micro-organismos patogênicos em um ambiente fisiologicamente asséptico e migração de bactérias para corrente sanguínea resultando em quadro de endotoxemia. A endotoxemia é resultado da ativação da cascata da inflamação em resposta aos componentes da parede celular de bactérias Gram-negativas. No caso em estudo houve contaminação do trato reprodutivo por bactérias patogênicas seguido de seu extravasamento para cavidade abdominal, local onde houve proliferação e lise bacteriana. A terapia instituída a base de fluidoterapia, antiinflamatórios e antibióticos teve como intuito o controle no processo endotoxêmico, no entanto, devido à retroalimentação do ciclo, sem remoção da causa primária, não foi observada resposta a terapêutica, resultando em óbito por peritonite e endotoxemia. A endotoxemia também foi resultado da severa metrite que tornou a parede uterina fina, friável e necrótica, resultando em absorção de bactérias e toxinas para circulação sanguínea sistêmica (BLANCHARD et al., 2010). 


\section{CONCLUSÃO}

A obesidade é sugerida como um fator de risco a ocorrência de distocia, sendo comum o sobrepeso em éguas criadas no estado do Rio Grande do Sul por questões culturais. A distocia seguida por retenção placentária em uma égua obesa induziu alterações sistêmicas que culminaram em ruptura uterina e extravasamento de conteúdo uterino para cavidade abdominal, sendo de difícil tratamento, evoluindo a óbito por peritonite e endotoxemia.

\section{PLACENTAL RETENTION AND UTERINE LACERATION IN MARE - CASE REPORT}

\section{ABSTRACT}

$\mathrm{P}$ lacental retention is associated with uterine inertia, and may cause systemic alterations. Uterine rupture induces the contamination of the abdominal cavity, and peritonitis, worsening clinical signs and risk for the animal's life. The aim of this paper is to report a case of retained placenta and uterine rupture in a mare. An obese mare was attended 5 days after it had experienced distocia. Tachycardia, tachypnea, congestion of mucosae, fever and retained placenta were observed. In the ultrasound examination, the uterus was distended and uterine wall was thickened, with anechoic content and hyperechoic spots in the lumen. The blood count showed leukocytosis, neutrophilia, lymphopenia and immature lymphocytes. Abdominal paracentesis was unproductive. Treatment was based on flunixin meglumine $1.1 \mathrm{mg} / \mathrm{kg}$, penicillin G potassium $20.000 \mathrm{UI} / \mathrm{kg}$, gentamicin $6.6 \mathrm{mg} / \mathrm{kg}$, oxytocin $20 \mathrm{UI}$, omeprazole $2 \mathrm{mg} / \mathrm{kg}$, heparin $60 \mathrm{UI} / \mathrm{kg}$ and uterine wash. After 3 days of treatment, clinical condition got worse and the animal died. In the necropsy, severe edema in the subcutaneous abdominal region, fatty liver, gastric ulcers, severe fibrin deposition in the abdominal cavity, uterine rupture with placental segment protrusion into the abdominal cavity, endometrial necrosis and presence of autolytic placental fragments were observed. Obesity might be considered a risk factor for the occurrence of dystocia. Placental retention favored the occurrence of uterine rupture, resulting in the release of uterine contents into the abdominal cavity, evolving to death due to peritonitis and endotoxemia.

Keywords: Birth. Dystocia. Endotoxemia. Puerperium. 


\section{RETENCIÓN DE PLACENTA Y RUPTURA UTERINA EN YEGUA - INFORME DE CASO}

\section{RESUMEN}

a retención de la placenta esta relacionada la inercia uterina, lo que puede causar cambios sistémicos. La ruptura uterina provoca contaminación de la cavidad abdominal, induce la aparición de peritonitis, empeorando los síntomas, teniendo riesgo de vida el animal. El objetivo de este trabajo es presentar un caso de retención de la placenta y ruptura uterina en una yegua. Una yegua, obesa, 5 días después de un parto distócico, presentó en el examen clínico taquicardia, taquipnea, congestión de las mucosas, fiebre y retención de placenta. En la ecografía, el útero estaba distendido, con engrosamiento de la pared uterina, contenido anecoico y puntos hiperecoicos en la luz. En el hemograma se encontró leucocitosis, neutrofilia, linfopenia y presencia de linfocitos inmaduros. La paracentesis abdominal fue improductiva. El tratamiento se inició con flunixina de meglumine $1,1 \mathrm{mg} / \mathrm{kg}$, penicilina $\mathrm{G}$ potásica $20.000 \mathrm{UI} / \mathrm{kg}$, gentamicina 6,6 $\mathrm{mg} / \mathrm{kg}$, oxitocina $20 \mathrm{UI}$, omeprazol $2 \mathrm{mg} / \mathrm{kg}$, heparina $60 \mathrm{UI} / \mathrm{kg}$ y lavado uterino. Después de 3 días de tratamiento, la condición clínica se deterioró y el animal murió. En la necropsia se mostro edema marcado de la región abdominal subcutánea, hígado graso, úlceras gástricas, deposición severa de fibrina en la cavidad abdominal, ruptura uterina con profusión de un segmento de la placenta para la cavidad abdominal, necrosis del endometrio y presencia de fragmentos de placenta en autolisis. La obesidad se ha sugerido como un factor de riesgo para la ocurrencia de distocia. La retención de la placenta favoreció la ocurrencia de ruptura uterina, proporcionando la extravasación del contenido uterino a la cavidad abdominal, de difícil tratamiento, lo que lleva a la muerte por peritonitis y endotoxemia.

Palabras clave: Distocia. Endotoxemia. Parto. Puerperio.

\section{REFERÊNCIAS}

ALVES, M. N. R. Os efeitos da obesidade na resposta imune. Revista Brasileira de Nutrição e Clínica, v. 21, n. 4, p. 316-319, 2006.

BLANCHARD, T. L.; MACPHERSON, M.L. Postparturient abnormalities. In: BLANCHARD, T. L.; VARNER, D. D.; LOVE, C. C. et al. Manual of Equine Reproduction. 3a ed. Mosby Elsevier, 2010. Cap. 10, p. 131-142.

BRINSKO, S. P. How to perform uterine lavage: indications and practical techniques. In: PROCEEDINGS OF THE 47TH AAEP ANNUAL CONVENTION, 47, 2001, San Diego, California, USA. PROCEEDINGS. San Diego, 2001, November, n. 24-28, p. 407-411. 
CARRASCOSA, J. M.; ROCAMORA, V.; FERNANDEZ-TORRES, R. M.; JIMENEZ-PUYA, R.; MORENO, J. C.; COLL-PUIGSERVER, N.; FONSECA, E. Obesity and Psoriasis: Inflammatory Nature of Obesity, Relationship Between Psoriasis and Obesity, and Therapeutic Implications. Actas Dermosifiliograficas, v. 105, n. 1, p. 31-44, 2014.

CHRISTENSEN, B. W. Parturition. In: MCKINNON, A. O.; SQUIRES, E. L.; VAALA, W. E.; et al. Equine Reproduction. 2a ed. Wiley-Blackwell, 2011. Cap. 233, p. 2268-2276.

CHRISTOFFERSSON M. Shoulder dystocia and brachial plexus injury: A population-based study. Gynecologic and Obstetric Investigation, v. 53, p. 42-44, 2002.

CUERVO-ARANGO J.; NEWCOMBE, J. R. The effect of manual removal of placenta immediately after foaling on subsequent fertility parameters in the mare. Journal of Equine Veterinary Science, v. 29, n. 11, p. 771-774, 2009.

CONWAY, B.; RENÉ, A. Obesity as a disease: No lightweight matter. Obesity Reviews, v. 5, n. 3, p. 145-51, 2004.

DOLENTE, B. A. Critical peripartum disease in the mare. Veterinary Clinics Equine, v. 20 n. 1, p. 151-165, 2004.

EL-WAKKAD, A.; HASSAN-NEL, M.; SIBAIl, H.; EL-ZAYAT, S. R. Proinflammatory, antiinflammatory cytokines and adiponkines in students with central obesity. Cytokine, v. 61, n. 2, p. 682-687, 2013.

FISCHER, A. T.; PHILIPS, T. N. Surgical repair of a ruptured uterus in five mares. Equine Veterinary Journal, v. 18, n. 2, p. 153-5, 1986.

GRONDIN, T. M.; DEWITT, S. F. Normal hematology of the horse and donkey. In: WARDRUP, J.; WEISS, D. Schalm's Veterinary Hematology. 6a ed. Wiley-Blackwell, 2010. Cap.106, p. 821-828.

HANSON, R. R. How I treat horses with peritonitis. Compendium Continued Education Practice Veterinary, v. 21, n. 10, p. 965-973, 1999.

HENNEKE, D. R., POTTER, G. D., KRIEDER, J. L. et al. Relationship between condition score, physical measurements and body fat percentage in mares. Equine Veterinary Journal, v. 15, p. 371-372, 1983.

HORTA, A. E. M. Etiopatogenia e terapêutica da retenção placentária nos bovinos. In: JORNADAS INTERNACIONALES DE REPRODUCCIÓN ANIMAL, 7, 2000, Murcia. ANAIS. AERA, Murcia, 2000, p. 181-192. 
HUDSON, N. P. H., PRINCE, D. R., MAYHEW, I. G.; et al. Investigation and management of a cluster of cases of equine retained fetal membranes in Highland ponies. The Veterinary Record, v. 157, n. 3, p. 85-89, 2005.

JOHNSON, C. A. Pregnancy management in the bitch. Theriogenology, v. 70, n. 9, p. 14121417, 2008.

JÚNIOR, J. O. L.; SERRANO, S. C.; TEODORO, A. L.; et al. Os antiinflamatórios não hormonais. Prática hospitalar, v. 51, p. 173-178, 2007.

KEARNS, C. F.; MCKEEVER, K. H.; ABE, T. Overview of horse body composition and muscle architecture: implications for performance. Veterinary Journal, v. 164, n. 3, p. 224-234, 2002.

LE BLANC, M. M.; NEUWIRTH, L.; ASBURY, A. C. Scintigraphic measurement of uterine cleareance in normal mares and mares with recurrent endometritis. Equine Veterinary Journal, v. 26, n. 2, p. 109-113, 1994.

MESSER, N. T. The use of laboratory tests in equine practice. Veterinary Clinical North American: Equine Pratice, v. 11, n. 3, p. 345-350, 1995.

PROVENCHER, R.; THRELFALL, W. R.; MURDICK, P.W. et al. Retained fetal membranes in the mare: a retrospective study. Canadian Veterinary Journal, v. 29, n. 11, p. 903-910, 1988.

ROSSDALE, P. D. The horse, from conception to maturity. 2a ed. London: Acribia S. A., 1993, $224 p$.

RUGE, S.; ANDERSEN, T. Obstetric risks in obesity: An analysis of the literature. Obstetrical \& Gynecological Survey, v. 40, p. 57-60, 1985.

SUTTER, W. W.; HOPPER, R. M.; FRAZER, G. S. Diagnosis and Surgical Treatment of Uterine Lacerations in Mares (33 Cases). In: ANNUAL CONVENTION OF THE AMERICAN ASSOCIATION OF EQUINE PRACTITIONERS, 49, 2003, New Orleans, Louisiana, USA. PROCCEDINGS. New Orleans, 2003. p. 357-359.

TIBARY, A.; DIP, A. C. T.; PEARSON, D. V. M. Distocia and obstetrical manipulations in the mare. In: CONFERÊNCIA ANUAL DA ABRAVEQ, 13, 2012, Campinas, SP. RESUMOS. Campinas, 2012, p. 213.

THRELFALL, W. R. Retained fetal membranes. In: YOUNGQUIST, R. S.; THRELFALL, W.R. Current Therapy in Large Animal Theriogenology, Philadelphia: Saunders-Elsevier, 2007. Cap. 12, p. 107-113.

TULLENERS, E. P. Complications of abdominocentesis in the horse. Journal American Veterinary Medicine Association, v. 182, n. 3, p. 232-234, 1983. 
TURNER, R. M. Post-Partum Problems: The Top Ten List. In: ANNUAL CONVENTION OF THE AMERICAN ASSOCIATION OF EQUINE PRACTITIONERS, 53, 2007, Orlando, Florida, USA. PROCEEDINGS. Orlando, 2007, p. 305-319.

VANDEPLASSCHE, M.; SPINCEMAILLE, J.; BOUTERS, R.; et al. Observations on involution and puerperal endometritis in mares. Irish Veterinary Journal, v. 37, n. 9/12, p. 126-132, 1983.

ZIMMERMAN, R. A.; GREENE, D. E. Caloric allowance Amett DW, Holland GL, Totusek R: Some effects of obesity in beef females. Journal of Animal Science, v. 33, p. 1129-1136, 1971.

ZORAN, D. L. Obesity in dogs and cats: a metabolic and endocrine disorder. Veterinary Clinics of North America Small Animal Practice, v. 40, n. 2, p. 221-239, 2010.

Autor para correspondência: Fernanda Carlini Cunha dos Santos. Departamento de Clínicas Veterinária, Faculdade de Veterinária, Universidade Federal de Pelotas. Campus Universitário s/n, Caixa Postal 354, CEP 96010-900, Pelotas, Rio Grande do Sul, Brasil. carlini@portoweb.com.br 